\title{
Mitochondrial DNA, diabetes and pancreatic pathology in Kearns-Sayre syndrome
}

\author{
J. Poulton ${ }^{1}$, S. O'Rahilly ${ }^{2}$, K. J.Morten ${ }^{1}$, A. Clark ${ }^{3}$ \\ ${ }^{1}$ Department of Paediatrics, John Radcliffe Hospital, Oxford, UK \\ ${ }^{2}$ Departments of Medicine and Clinical Biochemistry, Addenbrooke's Hospital, Cambridge, UK \\ ${ }^{3}$ Diabetes Research Laboratories, Radcliffe Infirmary, Oxford, UK
}

Summary Mitochondrial DNA (mtDNA) mutations are associated with diabetes mellitus but their role in the onset of hyperglycaemia is unclear. A patient presented with diabetes requiring insulin therapy at the age of 7 years, followed by diagnosis of KearnsSayre syndrome (KSS). Beta-cell function was absent at age 19 years as shown by lack of glucose-stimulated C-peptide secretion. Following development of a cardiac conduction defect the patient died aged 21 years. Analysis of mtDNA in blood and several tissues revealed related re-arranged deletions, duplications and deletion dimers in addition to normal mtDNA with the highest levels of duplications in kidney and blood. Pancreatic tissue from the KSS patient was compared with tissue from an insulin-dependent diabetic patient with a similar clinical history of diabetes. Islets in KSS were small, regular in shape and contained predominantly glucagon-containing cells with no evidence of beta cells. In comparison, a small number of beta cells were present in some of the larger more irregularly-shaped islets from the insulin-dependent diabetic patient. These data together suggest that in KSS the loss of beta cells at the onset of diabetes is less disruptive to islet architecture: a small proportion of beta cells or their gradual destruction over a long period would allow retention of islet shape. Abnormal function of the re-arranged mtDNA could affect both development and function of pancreatic islet cells since glucosestimulated insulin secretion is energy dependent. [Diabetologia (1995) 38: 868-871].

Key words Mitochondrial DNA, pancreatic islets, diabetes, Kearns-Sayre syndrome.
Mutations in mitochondrial genes have been implicated in some forms of maternally inherited diabetes mellitus $[1,2]$ and are associated with sporadic cases of both insulin-dependent (IDDM) and noninsulin-dependent diabetes (NIDDM) [3, 4]. Mitochondrial myopathies, including Kearns-Sayre syndrome (KSS), are a heterogenous group of disorders characterised by clumps of proliferated mito-

Received: 19 January 1995 and in revised form: 17 March 1995

Corresponding author: Dr. J. Poulton, Department of Paediatrics, John Radcliffe Hospital, Headington, Oxford OX3 9DU, UK

Abbreviations: mtDNA, Mitochondrial DNA; KSS, KearnsSayre syndrome; IDDM, insulin-dependent diabetes mellitus; NIDDM, non-insulin-dependent diabetes mellitus; ICA, islet cell antibodies. chondria found in muscle biopsies which are associated with mitochondrial DNA (mtDNA) mutations [5].

Patients have variable proportions of mutant and normal mtDNA (heteroplasmy) in different tissues. Tissue with high energy requirements such as muscle, brain and heart are affected most frequently, but endocrinopathies and diabetes in particular are a common occurrence. This could be due to the ATP requirement for insulin secretion [6]. Two types of mtDNA mutations are closely associated with diabetes, namely major rearrangements $[2,4]$ and point mutations in the tRNA ${ }^{\text {leu }}$ region, the so-called 3243 mutation [1]. There is very little evidence to explain the role of mtDNA mutations in the onset and progression of diabetes. To examine the effects of mtDNA rearrangements on islet structure and function, data has been correlated for islet secretion, pan- 
Table 1. Proportion of the different recombinant forms of mtDNA in ten tissues from the KSS patient (on molecular basis)

\begin{tabular}{llcrc}
\hline Tissue & \%Normal & $\begin{array}{c}\text { \%Dupli- } \\
\text { cation }\end{array}$ & \%eletion & $\begin{array}{c}\text { \%Deletion } \\
\text { dimer }\end{array}$ \\
\hline Cerebral & 58 & 3 & 18 & 19 \\
cortex & & & & \\
Cerebellum & 67 & 10 & 15 & 8 \\
Heart & 87 & 6 & 7 & $<1$ \\
Muscle 1 & 80 & 10 & $<1$ & 9 \\
Muscle 2 & 52 & 1 & 3 & 44 \\
Liver & 34 & 1 & 62 & 2 \\
Kidney & 30 & 16 & 36 & 18 \\
Pituitary & 55 & 11 & 23 & 11 \\
Pancreas & 59 & 10 & 39 & 3 \\
Ovary & 84 & 8 & 3 & 5 \\
Spleen & 65 & 7 & 18 & 9 \\
\hline
\end{tabular}

All these samples were taken at post-mortem except muscle 1 which was a biopsy taken 10 years prior to death

creatic histology and mitochondrial re-arrangements in a patient with Kearns-Sayre syndrome.

\section{Patients and methods}

Case history. This female patient presented at the age of 8 years with a 6-month history of lethargy, weight loss, polyuria and polydypsia. She was treated with insulin and, apart from occasional episodes of ketosis, her diabetes remained stable. She also developed night-blindness due to a pigmentory degeneration of the retina, external opthalmoplegia and ptosis. Muscle biopsy showed increased variation in fibre diameter with scattered ragged red fibres and abnormal mitochondria on electron microscopy. Mitochondrial myopathy was diagnosed and she then developed deafness, cerebellar ataxia and limb weakness. At age 19 years, an intraventricular conduction defect was detected by ECG and symmetrical low attenuations in the thalami were observed on computerized tomography (CT) scan. No islet cell antibodies could be detected and absence of C-peptide on glucose infusion confirmed her complete insulin dependence. She died aged 20 years, possibly due to a cardiac dysrhythmia in the presence of hypokalaemia caused by a renal potassium leak.

The MtDNA was analysed as previously described [7]. Briefly, whole-cell DNA was extracted from ten different tissues (Table 1), digested with Bam H1 and separated by electrophoresis for Southern blotting. Mitochondrial re-arrangements were identified with probes prepared from total purified mtDNA or regional probes cloned into bacteriophage M13 (courtesy of Dr. M. King (Columbia) and Professor G. Attardi (Caltech). The various forms of mtDNA were quantified by phosphor-image analysis.

Histological analysis. Post-mortem specimens of pancreas from the patient with KSS and a patient with clinically characterised IDDM (Patient T) were fixed in $10 \%$ formaldehyde in $0.9 \%$ $\mathrm{NaCl}$, dehydrated and embedded in wax for light microscopy. (Patient T presented at the age of 7 years with hyperglycaemia and islet cell antibodies, received insulin therapy and died at the age of 23 years from a road traffic accident). Sections were immunoperoxidase labelled with antisera specific for insulin, glucagon, pancreatic polypeptide, and somatostatin. Sec- tions immunolabelled for (a) glucagon and (b) insulin were semi-quantitatively assessed for (a) islet shape and size relative to a microscope eyepiece graticule and (b) the number of islets containing immunoreactivity to insulin, respectively. At least 50 islets were examined in each histological section.

\section{Results and discussion}

Three closely-related types of re-arranged mtDNA, in addition to the wild type were identified in the KSS patient: duplications, deletion monomers and deletion dimers. The proportions of each form varied in different tissues (Table 1 ) with the highest percentages of liver, deletion being found in the kidney and pancreas. In common with other cases of diabetes associated with mtDNA rearrangements this patient presented with insulin-requiring diabetes at an early age and glucose-tolerance testing showed that there was no residual beta-cell function 12 years after diagnosis. This is in contrast to the variable degrees of beta-cell function that are found in patients with $3243 \mathrm{mtDNA}$ mutations associated with IDDM or NIDDM although there is evidence for a declining insulin secretory capacity and a progression towards insulin dependence in these patients over a period of years $[3,8]$. Muscle biopsy samples taken 10 years apart (muscles 1 and 2 in Table 1 ) showed an increase in deletion dimers and reduction in duplications. Changes in the proportions of re-arranged mtDNA in muscle [7] and perhaps other tissues probably result in the evolving KSS phenotype. This increase in re-arranged mtDNA may be exacerbated by generating variable mtDNA mutations due to age-related accumulation of damage due to free radicals and lack of repair of mitochondrial genes. These could contribute to the heterogeneity of the onset and course of diabetes associated with mitochondrial mutations.

The histological appearance of the islets in the patient with KSS showed a complete absence of beta cells concomitant with lack of C-peptide secretion. Approximately $10 \%$ of islets from the IDDM patient contained at least one cell immunoreactive for insulin. Islets in both patients contained a high proportion of glucagon-containing cells. The endocrine cells in the KSS patient were retained within well-defined islet boundaries (Fig. 1a). In contrast, islets in patient $\mathrm{T}$ had the irregular shape which is typical of IDDM with glucagon-containing cells scattered between the exocrine tissue (Fig. 1b). This suggests that, in IDDM, the islet structure collapses when the beta cells are destroyed during the autoimmune phase of the disease, but islets in KSS do not collapse and a more regular architecture is retained.

The events leading to diabetes in KSS are unknown. Since this patient did not present with hyperglycaemia until the age of 7 years adequate insulin was available to maintain homeostasis until that time. It is likely that the beta cells originally formed a 

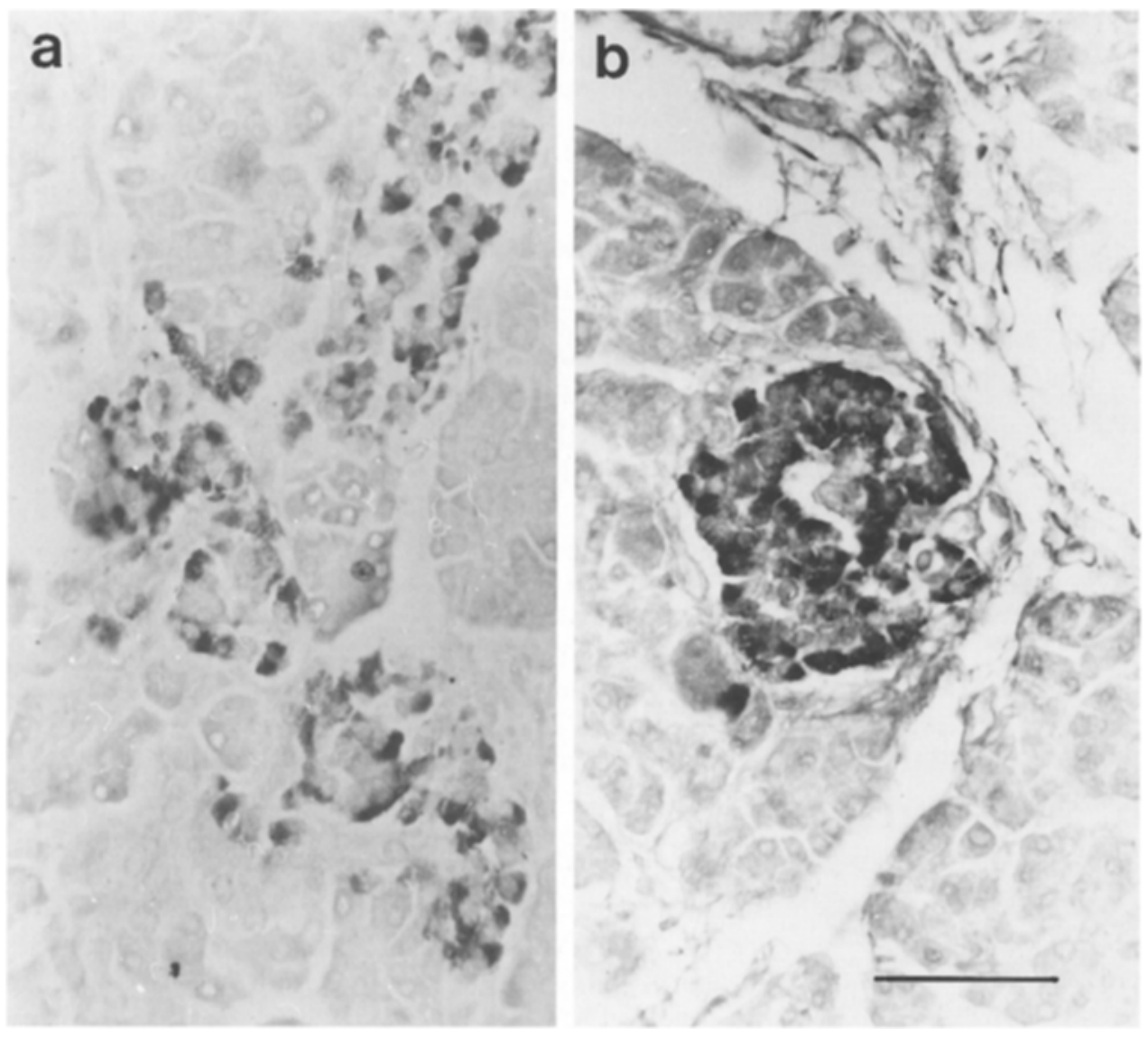

Fig.1a,b. Islets from an IDDM patient (a), and a KSS patient (b), immunoperoxidase labelled for glucagon. Islet architecture is disrupted in IDDM and glucagon (and other non-beta cells) are dispersed between exocrine acinar cells. The islets in KSS are small and compact. Islet profiles were significantly smaller in the KSS patient (mean $4.160 \mu \mathrm{m}^{2}$ ) compared to the IDDM patient (mean $\left.12.160 \mu \mathrm{m}^{2}\right)(p<0.001)$. Adjacent histological sections labelled for insulin, somatostatin and pancreatic polypeptide (not shown) demonstrated that $\alpha$-cells are the predominant cell type in both patients and no immunoreactive beta cells were detected in any of the 67 islet profiles examined from the KSS patient. Scale bar $=50 \mu \mathrm{m}$

smaller proportion of the islet structure than is found in normal subjects and/or were lost more gradually compared to the relatively acute phase of destruction of IDDM. There was no evidence for autoimmune diabetes in KSS: there was no inflammatory infiltrate in pancreatic islets or serum islet cell antibodies (ICA). Three patients with 3243 mutation, low titres of ICA, and HLA markers consistent with a susceptibility for IDDM have been identified [9].

Islets in KSS had a smaller cross-sectional area assessed by semi-quantitative methods compared to pancreatic islets in IDDM. Similar small islets have been identified in pancreatic specimens from diabetic patients with the 3243 mutation (Kadowaki, unpublished observations). Mutations in mtDNA are likely to contribute to islet development and function in the fetus which would result in lower numbers of islet cells in patients with abnormalities of mtDNA. Respiratory capacity is impaired in muscle cells containing high levels of either the 3243 mtDNA mutation or mtDNA deletions [5]. Alterations in mitochondrial function are likely to impair insulin secretion since glucose-stimulated insulin secretion is mediated by closure of ATP-dependent potassium channels in the beta-cell membrane [6]. Thus, insulin-secretion is dependent upon oxidative phosphorylation and intracellular ATP production. It has been suggested that fetal malnutrition is associated with impaired insulin secretion in adult life [10]. Our data suggest that the final common path determining beta-cell mass might be ATP and hence nutrient availability during a critical period of fetal development. Inadequate energy supply for insulin secretion could be a major susceptibility factor for development of both IDDM and NIDDM.

Acknowledgements. We are grateful to M.S.Chargé, Ms. M. Burnett and Ms. M.Deadman for expert technical assistance and to the British Diabetic Association and the Wellcome Trust for financial help. We would like to thank Professor E. Moxon for his continuing support and encouragement. We are indebted to the patient's family and to Dr. J.Wilson for permission to examine post-mortem material.

\section{References}

1. van den Ouweland JM, Lemkes HH, Ruitenbeek W, et al. (1992) Mutation in mitochondrial tRNA(Leu)(UUR) gene in a large pedigree with maternally transmitted type II diabetes mellitus and deafness. Nat Genet 1: 368-371

2. Ballinger SW, Shoffner JM, Hedaya EV, et al. (1992) Maternally transmitted diabetes and deafness associated with a $10.4 \mathrm{~kb}$ mitochondrial DNA deletion. Nat Genet 1: 11-15

3. Otabe S, Sakura H, Shimokawa K, et al. (1994) The high prevalence of the diabetic patients with a mutation in the mitochondrial gene in Japan. J Clin Endocrinol Metab 79: 768-771 
4. Poulton J, Morten K, Brown G, Bindoff L (1994) Are duplications of mitochondrial DNA characteristic of KearnsSayre syndrome? Hum Mol Genet 3: 947-951

5. De Vivo DC (1993) The expanding clinical spectrum of mitochondrial diseases. Brain Dev 15: 1-22

6. Ashcroft E, Ashcroft S (1992) Mechanisms of insulin secretion. In: Ashcroft F, Ashcroft S, (eds). Insulin: molecular biology to pathology. Oxford University Press Oxford pp 97150

7. Poulton J, Deadman ME, Bindoff L, Morten K, Land J, Brown $G$ (1993) Families of mtDNA re-arrangements can be detected in patients with mtDNA deletions: duplica- tions may be a transient intermediate form. Hum Mol Genet 2: 23-30

8. Kadowaki T, Kadowaki H, Mori Y, et al. (1994) A subtype of diabetes mellitus associated with a mutation of mitochondrial DNA. N Engl J Med 330: 962-968

9. Oka Y, Katagiri H, Yazaki Y, Murase T, Kobayashi T (1993) Mitochondrial gene mutation in islet-cell-antibodypositive patients who were initially non-insulin-dependent diabetics. Lancet 342: 527-528

10. Phipps K, Barker DJ, Hales CN, Fall CH, Osmond C, Clark PM (1993) Fetal growth and impaired glucose tolerance in men and women. Diabetologia 36: 225-228 